\title{
Capacidades del estado y de la población víctima de violencia sociopolítica para la construcción de paz: una mirada en regiones de Sucre
}

\section{Capacities of the state and the population victim of socio-political violence for the construction of peace: a view in Sucre regions}

\author{
Blanca Pérez-Contreras, M.Sc. \\ Corporación Universitaria del Caribe, Sincelejo, Sucre, Colombia. Magíster en Educación: Sociología de la Educa- \\ ción, Socióloga. Correspondencia: blanca.perez@cecar.edu.co.
}

Recibido: 20-01-2017; Aceptado: 14-03-2017

\section{RESUMEN}

El artículo es producto de la investigación "Diagnóstico de las capacidades técnicas, administrativas y humanas de las instituciones que atienden población víctima de violencia sociopolítica en las regiones golfo de Morrosquillo y Sabanas, departamento de Sucre, año 2014 -2015". Las manifestaciones de este fenómeno sociopolítico se configuraron en amenazas, reclutamientos, desapariciones forzadas, masacres por incursión de grupos al margen de la ley, lo que generó daños psicológicos, sociales, económicos y desplazamiento de la población civil a lugares ajenos con pérdida sustancial de sus bienes materiales, sociales y espirituales. Estos hechos llevaron al gobierno a tomar la decisión política de conocer el conflicto armado, la violación de derechos humanos, la existencia del estado de cosas inconstitucionales y la necesidad de reparar a las víctimas sancionando varias leyes, entre ellas la 387 de 1997 y la 975 de 2005, las que al no surtir los efectos esperados dieron origen a la Ley 1448 de 2011, que en Colombia se constituye en el marco conceptual, metodológico y jurídico que guía la operatividad de la política pública y se dictan "medidas de asistencia, atención y reparación integral a las víctimas del conflicto armado interno y se establecen otras disposiciones". La investigación se enmarcó en un enfoque mixto de tipo descriptivo - interpretativo, las técnicas utilizadas fueron la entrevista semiestructurada y la observación participante en contextos de atención a víctimas. Los resultados muestran fortalezas y debilidades en el cumplimiento de las funciones institucionales que determina la Ley 1448, no obstante, se enfatiza en las debilidades técnico-administrativas y humanas dada la relevancia política y social que esta información reviste, para redimensionar el actuar del Estado y la institucionalidad, en procura a garantizar el restablecimiento de derechos y la atención integral de las víctimas, para aportar a la construcción de paz desde lo regional y socio comunitario.

Palabras clave: Capacidad institucional, víctimas, reparación integral, construcción de paz, violencia sociopolítica 
Pérez-Contreras. Capacidades del estado y de la población víctima de violencia

\section{ABSTRACT}

Article is a product of the research "diagnostic of human, administrative, and technical capacities of institutions that serve victims of socio-political violence in the Gulf of Morrosquillo bed linen and regions, Department of Sucre, year 2014 - 2015". The manifestations of this socio-political phenomenon is configured in threats, recruitment, disappearances, massacres by RAID groups on the fringes of the law, generating social, economic and psychological damage and displacement of the civilian population to other places with substantial loss of material, social and spiritual assets. These facts led the Government to take the political decision to recognize the armed conflict, violations of human rights, the existence of the State of things inconstitucionales and the need to repair the victims sanctioning several, including the 387 of 1997, and the 975 of 2005, which gave rise to the law not to refill the expected effects 1448 2011 in Colombia it is conceptual, methodological and legal framework that guides the operation of the public policy and enacting "measures of assistance, care and full reparation to the victims of the armed conflict internal and other provisions". The research was part of a mixed approach of descriptive - interpretive, the techniques used were participant observation in context of attention to victims and the semistructured interview. The results show strengths and weaknesses in the performance of the institutional functions that determines the Act 1448, however, emphasizes technical and human weaknesses due to the political and social relevance of this information is to resize the actions of the State and institutions, in order to ensure the restoration of rights and the comprehensive care of victims to contribute to the construction of peace from regional and community partner.

Keywords: Institutional capacity, victims, integral reparation, peace building, sociopolitical violence

\section{Introducción}

"Vivir en una sociedad sin conflictos es imposible, construir caminos para la paz y una sociedad amigable para todos y todas es el reto".

La violencia sociopolítica en el ámbito nacional ha desencadenado una de las problemáticas más acuciantes en la historia sociopolítica colombiana: "el desplazamiento forzado", que ha afectado a unos municipios más que a otros y donde miles de personas aterradas por los hechos ocurridos en diferentes zonas del país han tenido que dejar su lugar de origen, con incidencia en sus condiciones de vida. Según datos de la Corporación Vínculos (2008), entre 1985 y 2005, fueron desplazadas en Colombia 3.7 millones de personas, número que se ha venido incrementando años tras año.
Sobre el particular, la Oficina para la Coordinación de Asuntos Humanitarios de las Naciones Unidas en Colombia $(\mathrm{OCHA})$, informa que el número de desplazados llegó aproximadamente a 5.500 personas en ocasión de 20 eventos masivos ocurridos entre enero y febrero de 2012, comparado con los 18 desplazamientos masivos que se presentaron en el mismo periodo durante el 2011. Asimismo, la Consultoría para los Derechos Humanos y el Desplazamiento - CODHES, afirma que en el año 2013 el número de desplazados se incrementó en 219.405 personas (Codhes- Sisdhes; UARIV (2014, p.3), aunque la cifra podría ser mayor pues aún muchas personas no han presentado declaración de su condición. Este aumento en el número de desplazados, año tras año en el territorio nacional, se refleja en las cifras reportadas por la Unidad de Atención y Reparación Integral a Víctimas en el año 
2013, que ascienden a 5.962.611) y las registradas por el CODHES de 5.921.229 (Codhes- Sisdhes; UARIV (2014).

Por su parte, el informe general del Centro Nacional de Memoria Histórica (Capítulo 1, p: 76), afirma que:

1.116 municipios registraron expulsión de población civil como consecuencia del conflicto armado, lo que representa un $97 \%$ del territorio nacional. En los casos más críticos, 139 municipios registraron más de 10.000 desplazados entre 1996 y 2012 , que equivale al $74 \%$ del total de la población desplazada. Entre estos, 57 registraron más de $20.000,26$ más de $30.000,12$ más de 40.000 y 9 más de 50.000 desplazados. También reporta 5.712.506 personas en condición de desplazamiento a 2013.

El departamento de Sucre, al igual que la mayoría de los departamentos de la costa Caribe y de Colombia, ha estado afectado por décadas por el flagelo de la violencia sociopolítica; grupos al margen de la ley han incursionado en las cinco subregiones que lo conforman, perpetrando masacres, reclutamiento forzado, muertes, hostigamiento y amenazas a la población civil. Durante el periodo 1997 a 2007 en la mayoría de municipios de Sucre predominó la disputa gobierno-guerrilla; el terror fue la estrategia más usada por estos grupos como mecanismo de guerra y doblegar la voluntad de las personas para conseguir sus fines. Es así, que en el año 1997, el $18 \%$ de los municipios fueron afectados, disminuyendo un poco su accionar entre los años 1998 a 2000, para ascender a su punto más crítico en el 2001 con un $30 \%$ de municipios afectados (las cifras de desplazados del año 2000 a 2001 ascendieron de 12.994 a 19.653 , coincidiendo este aumento con la arremetida y fortalecimiento del paramilitarismo ${ }^{1}$ - AUC - y los $1 \quad$ En Colombia el paramilitarismo hace referencia al fenómeno histórico relacionado con la acción de grupos armados ilegales de extrema derecha, organizados a partir de la década de los setenta para ayudar al gobierno a combatir a los grupos de extrema izquierda o guerrilla. Es una estrategia contrainsurgente que participó en el conflicto armado colombiano, siendo uno de los grupos que más víctimas ha dejado en el país. enfrentamientos de este grupo con la guerrilla ${ }^{2}$ ); disminuyendo para el 2005 a un $27 \%$. (Diagnostico departamental de Sucre, 2007: p. 4) y (Estudio realizado por la Misión de Observación Electoral (MOE) de Sucre y la Corporación Arco Iris $(2007$, p. 18).

La conflictividad guerrilla - gobierno fue determinante en la dinámica del desplazamiento forzado que afectó a las cinco (5) subregiones del departamento de Sucre, con mayor incidencia en Montes de María, golfo de Morrosquillo y Sabanas; la mayoría de sus municipios se convirtieron en expulsores 0 en receptores/expulsores de población desplazada.

La investigación se realizó en seis municipios: Corozal, Sincé y Sampúes ubicados en la región Sabanas; Tolú, Toluviejo y San Antonio de Palmito en golfo de Morrosquillo; la mayoría de las víctimas están asentadas en la zona urbana, provenientes unas del sector rural de los mismos municipios o de otros del departamento; en menor proporción están personas desplazadas de los departamentos de Bolívar, Córdoba, Magdalena y Cesar, ubicados en la costa Caribe y los del departamento de Antioquía.

Las víctimas producto de los hechos victimizantes han tenido que enfrentarse a condiciones y situaciones adversas como la pérdida de bienes materiales, el despojo de tierras, el abandono de empleos tradicionales, delitos contra la libertad e integridad sexual, hogares desechos, cambios en la estructura y composición familiar, así como graves secuelas psicológicas manifiestas en niños, niñas, adolescentes, hombres y mujeres.

En el departamento de Sucre, de acuerdo con la Red Nacional de Información (RNI), para octubre de

\footnotetext{
$2 \quad$ Formación militar no organizada en ejército que lucha por motivos políticos con el fin de imponer un determinado sistema político, económico y social en un lugar o país. En Colombia es un grupo armado o insurgente que actúa al margen de la ley y que utiliza tácticas de intimidación a la población civil y el Estado.
} 
Pérez-Contreras. Capacidades del estado y de la población víctima de violencia

2015 se encontraban inscritas en el Registro Único de Víctimas (RUV) 273.918 personas, es decir, el $32 \%$ del total de la población sucreña está en esta condición. En este informe se afirma que el grupo etario más afectado son mujeres en edades entre 24 a 35 años, y las niñas y adolescentes entre 0 a 17 años, lo que representa un $32 \%$ de las víctimas declarantes; respecto al total de declarantes masculinos el $35 \%$ son niños y adolescentes. Esto, sin considerar que persiste el subregistro según lo afirmaron los funcionarios de las instituciones que atienden víctimas en los respectivos municipios y lo ratificado por la representante de la procuradora regional de Sucre, Margarita Sarmiento Barragán (2016), quien al respecto indicó:

...por fin en el departamento de Sucre se empieza por el principio, "no sabemos, no conocemos hoy cuáles son las víctimas de nuestro departamento, lo único que se tiene es el informe unificado de víctimas que es global y, ahora hay una buena noticia en cuanto a que existirá un registro municipal de todas las víctimas, lo que celebramos porque es lamentable que en una problemática tan grande que tiene el departamento de Sucre, no se sepa cuáles son las víctimas y dónde están". Agregó además "hay un subregistro de víctimas, se tiene que actualizar la información en los municipios porque así lo exige la Ley y lo necesitan las víctimas y la población más vulnerable del departamento".

Han transcurrido más de cinco (5) décadas de conflicto sin que se tenga solución concreta a este problema, por el contrario, cada año se conocen nuevos reportes de desplazamiento en el ámbito nacional y departamental, lo que muestra la magnitud del problema que se vive en las regiones, departamentos y localidades de Colombia.

Conexo a lo anterior, se resalta que en Colombia la atención y reparación integral a las víctimas, así como las medidas para la prevención del desplazamiento siguen presentando enormes vacíos, lo que deja entrever que el Estado no ha asumido eficazmente su responsabilidad; se continúan presentando en una serie de cosas inconstitucionales, expresadas en que, durante los últimos 10 años, que son también los de la promulgación de la Sentencia T-025 de 2004, en promedio 280.723 desplazados siguen Ilegando a diversos rincones del país (CODHES, 2013, p. 3).

Las Leyes 387 de 1997 y la 975 de 2005, promulgadas con la intención de contrarrestar el problema, presentaron falencias en su aplicabilidad, no surtieron los efectos esperados, por lo que se crea la Ley 1448 de 2011, a través de la cual se genera el marco conceptual, metodológico y jurídico que guía la operatividad de la política pública, se dictan "medidas de asistencia, atención y reparación integral a las víctimas del conflicto armado interno y se establecen otras disposiciones". Asimismo, contempla cuáles son las instituciones con responsabilidad en la atención a las víctimas, definiéndolas como "aquellas personas que individual o colectivamente hayan sufrido un daño por hechos ocurridos a partir del $1^{0} \mathrm{de}$ enero de 1985, como consecuencia de infracciones al Derecho Internacional Humanitario o de violaciones graves y manifiestas a las normas internacionales de Derechos Humanos, ocurridas con ocasión del conflicto armado interno".

La investigación asume el concepto de víctima propuesto en la Ley 1448, asimismo, el reconocido por los tratados y acuerdos internacionales, en los cuales se le define como:

...toda persona que haya sufrido daños individual y colectivamente, incluidas lesiones físicas o personal que se valoran en función de los riegos mentales, sufrimiento emocional, pérdidas económicas o menoscabo sustancial de derechos fundamentales, 
como consecuencia de acciones $u$ omisiones que constituyan una violación manifiesta de las normas internacionales o una violación grave del Derecho Internacional Humanitario", (Comisión colombiana de juristas, 2007, p. 25).

Para la comprensión de violencia sociopolítica se tomó el concepto de Carlos Sluzki, (1995, p. 2) citado por Arévalo, L. (2010) quien considera que "la violencia política, en cualquiera de sus muchas variantes, tiene un efecto devastador y de largo alcance en quienes han sido sus víctimas" $y$, el de la Organización Mundial de la SaludOMS que considera la violencia como:

... el uso intencionado de la fuerza física o del poder, sea como amenaza o como realidad contra uno mismo, contra otra persona o contra un grupo o comunidad, que tiene como resultado lesiones traumáticas, muerte, daños psicológicos, alteración del desarrollo o privaciones.

Los dos conceptos anteriores son relevantes en la medida que no solo hacen referencia a muertes $y$ lesiones traumáticas sino que además, incluyen daños psicológicos y todas las situaciones de privación ejercidas sobre una persona o una comunidad que comprometen su salud y su bienestar, lo que es determinante al momento de valorar los procesos de atención y reparación integral que brinda la institucionalidad en concordancia con lo que establece la Ley 1448, la que sirve de referente conceptual y metodológico en la investigación.

El objetivo de investigación se orientó a identificar las capacidades técnico/ administrativas y humanas en instituciones que atienden a la población víctima en seis municipios de las subregiones Sabanas y golfo de Morrosquillo, para lo cual se tuvo como referente aspectos tales como: existencia de base de datos sistematizada, rutas y protocolos para la atención, trabajo interinstitucional, trato humanizado y atención con enfoque diferencial, confidencialidad de los procesos, conocimiento y manejo de la ley, estrategias y mecanismos internos e interinstitucionales que se implementan en la atención de las víctimas y en la prevención de la re victimización, (Sistema de Corresponsabilidad, 2013).

\section{METODOLOGÍA}

En la investigación se utilizó un enfoque de complementariedad que permitió acceder a información cualitativa y cuantitativa en cuanto a los procesos de asistencia, atención y reparación integral a las víctimas. El tipo de investigación es descriptivo e interpretativo y las técnicas e instrumentos de recolección de información que se utilizaron fueron la entrevista semiestructurada y la observación en contexto; el procesamiento y análisis de la información se hizo a partir de las categorías estudiadas: Capacidades técnico/administrativas y humanas.

La información obtenida mediante la aplicación de la entrevista semiestructurada se ordenó de acuerdo con los patrones de respuestas obtenidas de los funcionarios en cuanto a capacidades institucionales, las que en su conjunto sirvieron de parámetro de comparación para determinar el cumplimiento o no de lo que establece la Ley 1448, (2011) en cuanto a "medidas de atención, asistencia y reparación integral a víctimas violencia sociopolítica. Por su parte, la observación en contexto sirvió como medida de verificación de las condiciones de infraestructura física, existencia de registros de información y dispositivos de comunicación y atención.

En la subregión golfo de Morrosquillo y Sabanas, se trabajó a nivel municipal con las instituciones a las que el Estado les atribuye funciones para la atención y reparación integral a víctimas y que aparecen asimismo referenciadas en los planes de desarrollo de los alcaldes: oficina de Enlace de Víctimas, 
la Personería Municipal, SENA, Banco Agrario, Secretaria de Educación, Registraduría Municipal, ESE municipal, Comisaria de Familia, Secretaria del Interior y Convivencia Ciudadana, Policía Nacional, Red Unidos, Secretaria de Salud, (Plan de Desarrollo Municipal, 2012-2015).

\section{RESULTADOS}

Los resultados se presentan por categorías, iniciando el análisis de los aspectos considerados en esta investigación para valorar, por una parte, el cumplimiento de la Ley 1448 en cuanto a capacidades técnico/ administrativas y por la otra, los referidos a las capacidades humanas.

Capacidades técnico/ administrativas. Respecto a la capacidad administrativa de mantener, sistematizar y actualizar información requerida en los procesos de atención y reparación integral, se verificó que la Unidad Administrativa Especial para la Atención y Reparación Integral a las Víctimas, ubicada en Sincelejo capital del departamento de Sucre, a 2015 no contaba con una base de datos actualizada sobre el número real y total de las víctimas en las subregiones, so pena que el artículo 154 de la Ley 1448 reza que es la responsable del "funcionamiento del Registro Único de Víctimas - RUV" y la que tiene la facultad de manejar una base de datos sistematizada y única, lo que es concordante con la información que aparece en otras fuentes mencionadas anteriormente.

En las subregiones estudiadas el registro y sistematización de la información varía en cada municipio: En San Antonio de Palmito la mayoría de las instituciones (70\%) cuenta con una base de datos de la población inscrita atendida e incluida en el Registro Único de Víctimas RUV, pero, esta no es abierta a otras instituciones; en Toluviejo y Santiago de Tolú el registro de la población desplazada se hace de forma sistematizada pero no está actualizado a 2015; en Corozal y Sincé, el $50 \%$ de las instituciones tienen la información sistematizada, pero no actualizada, en tanto que en Sampúes, la única institución con una base de datos organizada, actualizada y sistematizada es la oficina Enlace de Víctimas. Al respecto, la mayoría de los funcionarios afirmaron: no se tiene una caracterización actualizada de las víctimas; la información registrada corresponde a los años 2002 a 2010. En este sentido, la Corte Constitucional en el Auto 383 de 2010, señala como ejemplo de falta de capacidad administrativa, "la ausencia de sistematización de la información, la ineficiencia en la gestión de los recursos técnicos y humanos, la carencia de instrumentos adecuados de planificación", con afectaciones en una adecuada asistencia, atención y reparación integral.

Otra de las debilidades institucionales es la insuficiencia de dispositivos como el uso de protocolos y el manejo de rutas claras para la atención oportuna y óptima de la población víctima. En la región golfo de Morrosquillo la Personería Municipal y la Oficina de Enlace de víctimas cuentan con algunos protocolos mientras que en la región Sabanas la oficina Enlace de Víctimas es la única que cuenta con dispositivos de atención y reparación integral, que incluyen aspectos referidos a bienestar, seguridad, ayuda psicológica, económica, salud, educación, prevención, mecanismos de asistencia, protección integral y fortalecimiento institucional (ver figura 1 y 2 ).

Las demás instituciones brindan una atención poco directa a las víctimas, atienden a la población en general, y el trato que recibe la mayoría de las víctimas no es diferenciado, presentándose inconsistencia entre lo que determina la ley y lo que ocurre en la práctica. El incumplimiento en el manejo de protocolos y rutas vulnera el artículo 10 de la Ley 1448 que reza: "El Estado a través de las diferentes 
instituciones deberá promover mecanismos de publicidad eficaces, dirigidos a las víctimas". Es decir, a través de estos deberán brindar información y orientar a las víctimas acerca de los derechos, medidas y recursos con los que cuenta la institución, al igual que sobre los medios y rutas judiciales y administrativas a través de las cuales podrán acceder para el ejercicio de sus derechos en condiciones de dignidad.

\section{Protocolos de atención}

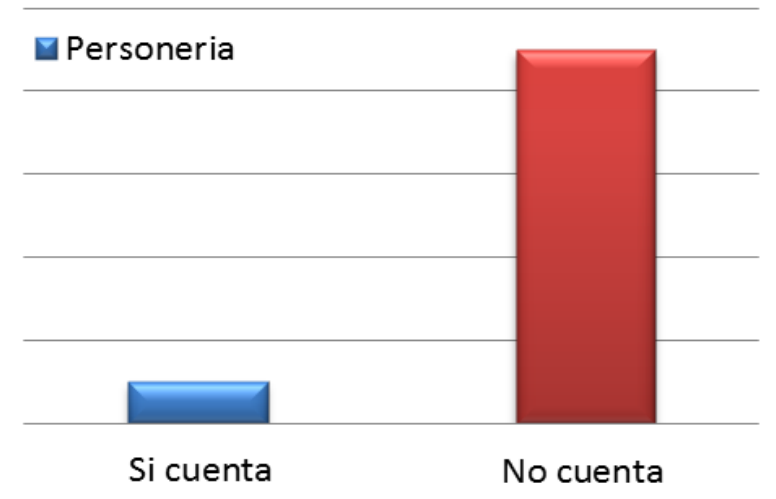

Figura 1. Manejo de protocolos

Fuente: Resultados entrevista aplicada a funcionarios, 2015

\section{Rutas de atención}

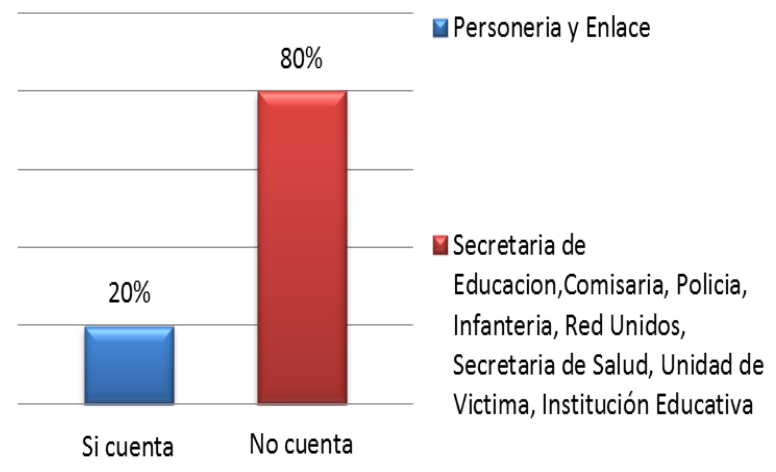

Figura 2: Existencias de rutas de atención a las víctimas

Fuente: Resultados entrevista aplicada a funcionarios, 2015

En cuanto a la capacidad administrativa "diseño de planes estratégicos y de acción de manera coordinada e interinstitucional" se pudo evidenciar que las instituciones en San Antonio de Palmito cuentan con planes, programas de atención y rehabilitación, pero, las medidas de atención en educación y el trabajo interinstitucional presentan falencias; además no cuentan con los recursos económicos y la organización suficiente para brindarle una mejor atención a esta población. (Entrevista a funcionarios, (2014). En la región Sabanas el mayor incumplimiento lo tienen las instituciones que operan en Sampúes, la única institución que cuenta con planes, medidas de rehabilitación, programas psicosociales dirigidos a todas las víctimas, tal como se define en el capítulo VIII de las medidas de satisfacción, artículo 135, 136 y 174 es la Oficina de Enlace.

En Corozal y Sincé, las instituciones no cuentan con planes de acción ni con estrategias de recuperación emocional a nivel grupal, indemnizaciones transformadoras, participación en banco de proyectos, ruta de retornos y reubicación individual. Ello indica, que muchas de las víctimas por desconocimiento no reciben los beneficios que proclama la ley en cuanto al "derecho a reparación integral a nivel comunitario o grupal.

El trabajo interinstitucional es otra de las principales falencias de la institucionalidad, a pesar de lo que señala el artículo 26 de la ley, no se hace un trabajo armónico y articulado, lo que impide una atención más efectiva y duradera. El Banco de Datos de Derechos Humanos y Violencia Política CINEP y la Red Nacional de Información, (2015: pp. 6), reporta que "la crisis humanitaria precedida por la falta de soluciones duraderas en los territorios hace que se encuentren miles de personas víctimas de desplazamientos en medio de emergencias prolongadas que hacen cuestionable su atención".

Los funcionarios consideraron, además, que uno de los obstáculos para mejorar la calidad de los servicios es la falta de apoyo suficiente de la Unidad de Atención y Reparación a Víctimas, la 
destinación de recursos es poca y no reciben capacitación en la Ley 1448. Sobre el aspecto financiero el estudio realizado a nivel nacional por Forero, E. (2010, p. 16) afirma que:

El Estado en su precariedad de recursos se ha limitado a tratar de atender el aspecto puramente asistencial relacionado con derechos sociales $y$ económicos: salud, educación, vivienda, tierras y generación de ingresos", lo cual ha obligado, por un lado, a las familias desplazadas a recurrir a la formulación de "tutelas" como el mecanismo para obtener el reconocimiento de todos sus derechos, y por otro, a la Corte Constitucional a declarar el "estado de cosa inconstitucional" en la situación de la población desplazada, Forero, E. (2010, p. 16).

En los resultados también se ponen de manifiesto que:

La falta de coherencia entre las obligaciones constitucionales y legales a cargo de las entidades públicas para atender y proteger los derechos de la población víctima de la violencia y lo recursos destinados a garantizar sus derechos fundamentales, ha generado traumatismos en el orden nacional, departamental y municipal, muestra la falta de capacidad institucional para dar respuesta efectiva a las necesidades de las víctimas, Forero, E. (2010, p. 16).

Respecto al tipo de ayuda que reciben las víctimas, los funcionarios informaron que esta es más de carácter inmediato (vivienda, salud y alimentación), lo que es considerado un paliativo frente a la complejidad de las necesidades que demanda esta población. Las ayudas de emergencia y transición no son otorgadas a las víctimas, a pesar de lo que determina la Ley 1448: "la asistencia y ayuda humanitaria son el conjunto de medidas orientadas a restablecer la vigencia efectiva de los derechos de las víctimas, brindarles condiciones para llevar una vida digna y garantizar su incorporación a la vida social, económica y política", (Ley 1448 de 2011).

Por su parte, el enfoque diferencial es poco aplicado en las instituciones de la región golfo de Morrosquillo y Sabanas. En Corozal y Sincé el $11 \%$ y $33 \%$ de las instituciones respectivamente, dicen aplicar lo que dispone la ley en materia de restitución de derechos por características particulares: edad, género, orientación sexual, grupo étnico y situación de discapacidad. No obstante, los porcentajes son mínimos, no son representativos de términos de una real aplicabilidad del enfoque diferencial a partir de las necesidades e intereses específicos; además, no existen los Planes Individuales de Reparación Integral a las víctimas.

En sí, aplican atención diferencial la Personería Municipal y la Unidad de Atención a Víctima, se aclara que esta última no tiene oficina en los municipios, solo un cogestor que hace el enlace con la Oficina Departamental (ver figura 3). Es posible entonces, que las instituciones que no se acogen a lo establecido en el artículo 13 de la Ley 1448 de 2011, incidan de manera directa o indirecta en aumentar el riesgo de discriminación y desigualdad en los procesos de atención. Esta situación se hace común a otros contextos, así queda expreso en relatos de víctimas que aparecen en las notas de campo del Centro de Memoria Histórica, (2013); en las continuas quejas y reclamos de las víctimas sobre los procesos de atención expresan: "en la atención hay irrespeto a la dignidad, no se tiene en cuenta la condición de género y edad". Información que sirve de fundamento para reafirmar que las falencias en la aplicabilidad de la ley, persisten. 


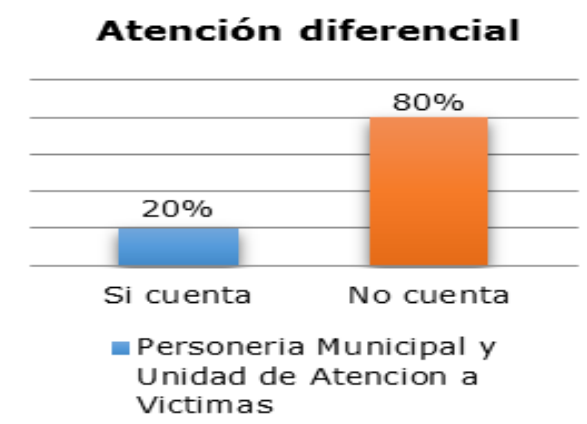

Figura 3: Aplicación del enfoque diferencial Fuente: Resultados entrevista aplicada a funcionarios, 2015

En cuanto a la implementación de estrategias de prevención de la re victimización, cinco instituciones de los municipios de Toluviejo, Tolú y San Antonio de Palmito - región Golfo de Morrosquillo - las Ilevan a cabo: la Personería Municipal, Policía Municipal, la Secretaria de Educación, Oficina de Enlace y la Comisaría de Familia, las que consisten en: a) informar y motivar a denunciar cualquier hecho que cause daño o atente contra sus derechos, b) la realización de visitas periódicas a los lugares de residencia por medio de charlas preventivas y c) participación en las mesas de infancia en la realización del concejo de política social (Ver figura 4). En los municipios de la región sabanas no fue posible acceder a esta información.

\section{Aplicación de estrategias}

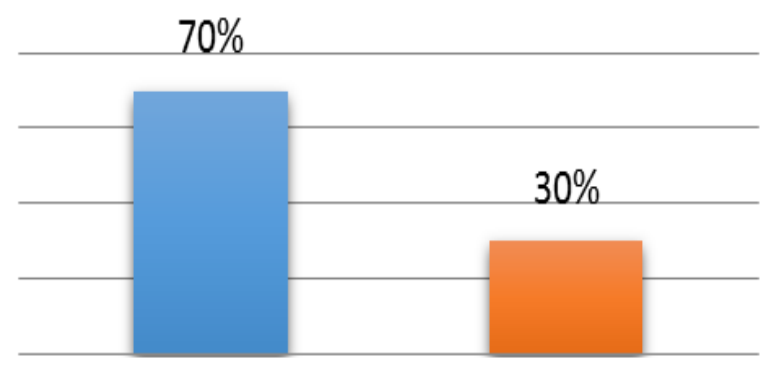

No cuentan

Figura 4: Prevención de re victimización Fuente: Resultados entrevista aplicada a funcionarios, 2015
Capacidades humanas. En cuanto a las capacidades humanas, el Estado colombiano incorpora el enfoque psicosocial en los procesos de asistencia, atención y reparación integral a las víctimas. Su puesta en marcha implica que las instituciones desarrollen políticas que garanticen una adecuada atención representada en: trato humanizado, confidencialidad en los procesos, conocimiento y manejo de la Ley 1448.

El estudio permitió acceder a información relevante al respecto. En la región golfo de Morrosquillo, los municipios de San Antonio de Palmito y Toluviejo el personal brinda atención inmediata; cumplen con el perfil, aunque el número que labora no es suficiente y las víctimas son tratadas con consideración, respeto y cordialidad, lo que guarda correspondencia con el artículo $4^{\circ}$. Dignidad. Artículo 15. Respeto mutuo. Artículo 178. Los funcionarios públicos deben tratar a las víctimas con humanidad y respeto de su dignidad y sus derechos humanos. Es un logro significativo que contribuye a avanzar en procesos de reconstrucción de tejido social y construcción de paz.

En Tolú, el personal no está capacitado para brindar una atención psicosocial, solo cuenta con acompañamiento jurídico, lo que controvierte el artículo 49 en cuanto a la acción de acompañamiento psicosocial a la víctima, con miras a facilitar el acceso y cualificar el ejercicio de los derechos a la verdad, justicia y reparación". Según (Sáenz, 2010), las vulneraciones vividas y el incumplimiento de la medida de reparación y atención en salud desde una perspectiva psicosocial por parte del Estado, de acuerdo con los resultados de la investigación realizada, reconfiguraron, "escenarios de desesperanza, dolor e impotencia y otros impactos psicosociales individuales y colectivos".

En la región Sabanas no todas las institucionesofrecen unacompañamiento 
Pérez-Contreras. Capacidades del estado y de la población víctima de violencia

psicosocial positivo. En Corozal, el procedimiento que sigue el $22 \%$ de las instituciones es la remisión de víctimas a otras instituciones en Sincelejo; Sincé el $11 \%$ brinda atención psicosocial, sin embargo, el personal que atiende no cuenta con las capacidades humanas para brindar atención integral. Se considera caso crítico lo que ocurre en la Personería Municipal de Sampués, donde atiende una secretaria y un abogado, no hay personal capacitado para brindar una óptima atención a la víctima, por ejemplo, si esta presentara una crisis al momento de la atención no se cuenta con profesionales de psicología o trabajo social, con ello, se puede generar re victimización. Estas falencias requieren atención por parte del Estado, ejercer una mayor vigilancia para garantizar la operatividad eficiente y efectiva de la ley.

En cuanto al conocimiento de la ley, el personal que trabaja en la oficina de Enlace de Víctimas, Personería Municipal, Comisaría de Familia, Unidad de Atención a Víctimas y Red Unidos tienen un gran manejo de esta, pero, no hacen visible estos conocimientos a la hora de atender a las víctimas. La Secretaría de Salud, Secretaría de Educación, las instituciones educativas, la policía e infantería de marina, afirmaron no tener conocimiento de la ley (ver figura 5). Ibáñez, A. y Vélez, C. (2003), consideraron que los municipios colombianos afrontan, por lo general, "restricciones de capital humano y tienen una capacidad institucional reducida lo cual complica aún más la provisión de asistencia a la población desplazada". A pesar de este vacío de conocimientos la capacitación de los funcionarios es mínima, lo que redunda negativamente en la atención integral a las víctimas e igualmente falta fortalecer el recurso humano interdisciplinario.
MANEJO DE LA LEY

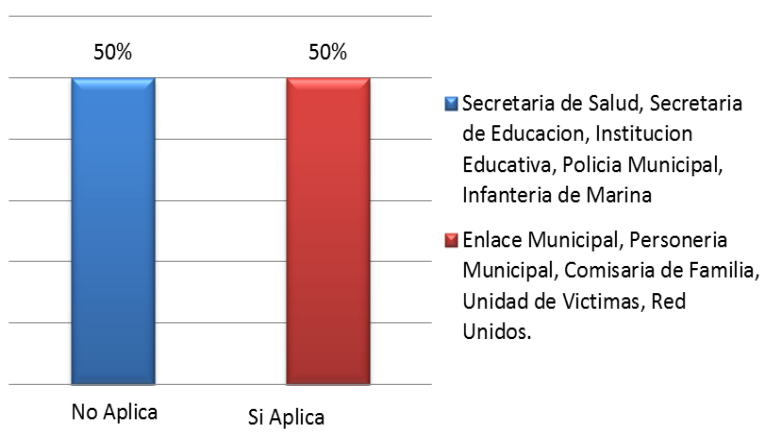

Figura 5: Conocimientos sobre la ley de víctimas Fuente: entrevista aplicada a encargados de la atención a víctimas, 2015

En lo referente a la garantía de confidencialidad, se observó que en la oficina de Enlace Municipal y la personería municipal atendían a varias víctimas al mismo tiempo, lo que está relacionado con las condiciones locativas. El principio de la confidencialidad en los procesos no se garantiza. Asimismo, las oficinas de la Secretaría de Educación y Salud están situadas en el mismo espacio y divididas por cubículos. En Toluviejo, la Comisaría de Familia en la fecha que se realizó el trabajo de campo no contaba con un lugar establecido para brindar atención a los usuarios, se ubicaba en instalaciones de la alcaldía municipal (ver figura 6).

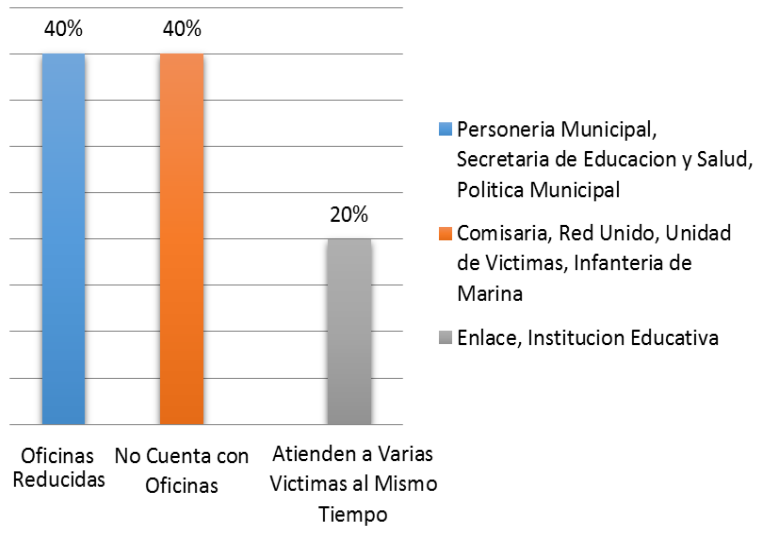

Figura 6: Confidencialidad.

Fuente: Entrevista realizada a funcionarios encargados de la atención víctimas, 2015. 
A modo de reflexión, se resalta el incumplimiento de la ley en materia de atención y reparación integral; los testimonios dados por algunas víctimas residentes en los municipios de la región Sabanas y golfo de Morrosquillo así lo dejan entrever, pues, en estos se devela su sentir e inconformidad, veamos: "los procesos de asistencia, atención y reparación no son ágiles ni oportunos, no recibimos información clara sobre el avance de los procesos" (visita domiciliaria, 2014). Si se comparan con los testimonios que se registran en la investigación realizada por Corporación Vínculos en convenio con el Programa de las Naciones Unidas para el Desarrollo - PNUD y otras organizaciones no gubernamentales especializadas, (2009), donde las víctimas expresan: "Necesitamos apoyo psicológico, que continúen esta valoración, porque si no, eso no va a ser un proceso igual que si vuelven dentro de un año, nada nos ganamos con que vengan hoy $y$ no vuelvan después", (Informe final, sentencia Ituango y Pueblo Bello, agosto de 2009), pareciera que el tiempo no hubiera pasado, se continúa con la insatisfacción sentida por muchas víctimas de la violencia sociopolítica.

\section{CONCLUSIONES}

Frente a todos esos sentires y demás falencias encontradas en la investigación la pregunta que todos y todas debemos hacernos es ¿Qué lectura se le está dando a la Ley 1448 por parte de la institucionalidad? ¿Cuál es la apuesta que permitiría transitar a un goce efectivo de los derechos de las víctimas? ¿Qué papel juegan las víctimas y el resto población civil en estos procesos?

En este contexto, los resultados pueden motivar reflexiones orientadas a una autoevaluación consciente del quehacer de las instituciones, para canalizar verdaderos cambios y de esta manera aportar a la restitución del tejido familiar, comunitario y social, creando las condiciones para una sociedad más saludable, justa, democrática, solidaria e inclusiva.

Se destaca que para minimizar las debilidades presentes en el sistema de atención y reparación a las víctimas el Estado necesita:

Transitar de una política de corte asistencialista hacia una política pública más comprometida con el desarrollo humano, fomentando la creación de redes solidarias y de apoyo comunal.

Reconocer la participación activa de las víctimas en proyectos que tomen en cuenta sus capacidades y habilidades personales, los recursos comunitarios para la autogestión del desarrollo humano y social.

La construcción de espacios de diálogo en los cuales las víctimas se sientan reconocidas como actores activos en la construcción de sus proyectos de vida.

Incentivar la comprensión y análisis de la realidad personal y comunitaria mediante escenarios de participación y construcción de nuevas realidades orientadas a la transformación social.

Promover comportamientos y actitudes resilientes como estrategia para alcanzar la reparación integral efectiva, la prevención de re victimización por indiferencia del Estado, el sistema jurídico y por estigmatización de la sociedad.

La formación de facilitadores locales que a su vez proporcionen formación, asesoría, en momentos difíciles y de crisis. 


\section{REFERENCIAS}

Alcaldía de Sampúes, (2012-2015). Plan para la atención integral a las víctimas de violencia sociopolítica y desplazamiento. Sampúes, Sucre. Recuperado de: http://www. sampues-sucre.gov.co/apc-aa-files/ 61 66643435626631653534373662343 3/plan-de-accion-2012-2015-listo2.pdf

Arévalo, L. (2010). Atención y reparación psicosocial en contextos de violencia sociopolítica: una mirada reflexiva. Revista de Estudios Sociales, no. 36, agosto de 2010, pp. 29. Universidad de Los Andes Bogotá, Colombia. Recuperado de: file:///c:/users/ cliente/desktop/rrr/-data-revista no. 36-03 dossier 02.pdf

Centro Nacional de Memoria Histórica. Informe General, (2013). Impactos $y$ daños del Conflicto Armado en Colombia, capítulo IV.

CINEP, (2015). Banco de Datos de Derechos Humanos y Violencia Política. Informe especial. Revista Noche y Niebla. Recuperado de: http:// webcache.googleusercontent. com/search?q=cache:http://www. nocheyniebla.org/

CODHES, (2012). Boletín de la Consultoría para los Derechos Humanos y el Desplazamiento, No. 79. Bogotá. Recuperado de: http://www.acnur.org/t3/uploads/ media/CODHES Informa 79 desplazamiento creciente $y$ crisis humanitaria invisibilizada marzo 2012.pdf?view $=1$

Comisión Colombiana de Juristas (2007). Principios internacionales sobre impunidad y reparaciones. Compilación de documentos de la Organización de Las Naciones Unidas Recuperado de: http:// www.coljuristas.org/documentos/ libros e informes/principios sobre impunidad y reparaciones. pdf

Consultoría para los Derechos Humanos y el Desplazamiento - CODHES, (2013). El Desplazamiento forzado y la imperiosa necesidad de la paz. Informe desplazamiento 2013. Recuperado: Cfr. http:// rni.unidadvictima.s.gov.co/?q=vreportes

Corte Constitucional. 2010. Sentencia T-045 de 2010 en respuesta a la acción de tutela instaurada por la Comisión Colombiana de Juristas en representación de Diana Carmenza Redondo, Argénida Torres, María Romero y Juana Cárdenas, contra el Ministerio de la Protección Social. Bogotá. Recuperado de: http:// www.corteconstitucional.gov.co/ relatoria/2010/T-045-10.htm

Departamento Administrativo Nacional de Estadística, DANE (2005). Censo General 2005, reporte generado en el 2014. Colombia. Recuperado de: http://www.dane.gov.co/files/ censos/Grupo mixto22\%20 PUBL. pdf

Diagnóstico Departamental Sucre (2007). Observatorio del Programa Presidencial de Derechos Humanos y Derecho Internacional Humanitario. p. 2.

Ejercicio de rendición social y pública de cuentas administradora Colombia (2012). Disponible en: $\quad$ http://rendircuentas. org/2013/04/corporacion-avreacompanamiento-psicosocialy-atencion-en-salud-mental-avictimas-de-violencia-politica2012-nacional-colombia/ 
El Espectador, (2014). "Miles de víctimas esperan indemnizaciones en Sucre". Redacción nacional. 16 de junio. Disponible en: http:// www.elespectador.com/noticias/ nacional/miles-de-victimasesperan-indemnizaciones-sucrearticulo-498686.

Ibáñez, A.M y Vélez, C. (2003). Instrumentos de atención de la población desplazada en Colombia: Una distribución desigual de las responsabilidades municipales. CEDE.

Informe departamental de hechos victimizantes, (2012). Departamento de Sucre. Recuperado de: http://rni.unidadvictimas.gov. co/sites/default/files/Documentos/ Sucre.pdf

Informe General Centro Nacional de Memoria Histórica. "Basta Ya", Colombia. Una Guerra prolongada y degradada. Dimensiones y modalidades de violencia, capítulo I, pp. 76. Recuperado de: http:// centrodememoriahistorica.gov.co/ descargas/informes2013/bastaYa/ capitulos/basta-ya-cap1 30-109. pdf

Informe sobre Desarrollo Humano 2011. (PNUD). Recuperado de: http:// hdr.undp.org/es/content/informesobre-desarrollo-humano-2011

Ley 1448 o Ley de Víctimas y Restitución de Tierras, (2011). Por la cual se dictan medidas de atención, asistencia y reparación integral a las víctimas. Congreso de la República de Colombia. Bogotá. Recuperado de: http://www. centrodememoriahistorica.gov. co/descargas/ley victimas/ley victimas completa web.pdf
Monografía Político Electoral DEPARTAMENTO DE SUCRE 1997 a 2007. Con el apoyo de misión de observación electoral (MOE). Corporación Nuevo Arco Iris. Recuperado de: http://moe.org. co/home/doc/moe mre/CD/PDF/ sucre.pdf

Panorama actual de la región de Montes de María y su entorno, (2003). Observatorio del Programa Presidencial de Derechos Humanos y Derecho Internacional Humanitario, p. 5. Recuperado de: http://www. acnur.org/t3/uploads/media/ COI 336.pdf?view $=1$

Panorama actual de Sucre, (2006). Observatorio del Programa Presidencial de Derechos Humanos y Derecho Internacional Humanitario. Recuperado de: http://historico. derechoshumanos. gov.co/Observatorio/Publicaciones/ documents/2010/Estu Regionales/ sucre.pdf

PlandeDesarrollodel Municipio deCorozal, (2012-2015). "Corozal, Ciudad del conocimiento". Recuperado de: http://www.corozal-sucre.gov.co/ apc-aa-files/ 373466636439316 63837336535626662/PLAN DE DESARROLLO 2012 2016.pdf

Plan de desarrollo del municipio de Sincé (2012-2015). "Hacia la prosperidad social". Recuperado de: http://www. since-sucre.gov.co/apc-aa-files/ 65633533623961646330616464 613533/PLAN DE DESARROLLO SINC $2012 \quad 2015$ LISTO.1 2.pdf

Plan de desarrollo departamental de Sucre, (2012-2015). "Acciones claras para dejar huellas". Recuperado de: file:///C:/Users/ cliente/Desktop/rrr/Plan de Desarrollo 2012-2015 Sucre.pdf 
Programa de atención psicosocial y salud integral a víctimas en el marco de la Ley 1448 de 2011. (2012). Versión 2 Ajustada. Elaborada por la Oficina de Promoción Social. Bogotá. Recuperado de: https://www. minsalud.gov.co/Documentos\%20 y\%20Publicaciones/PAPSIVI\%20 VERSION \% 20PRELIMINAR \% 20 25\%20Febrero\%202013.doc

Sáenz, M. (2010). Impacto del no cumplimiento de la medida de reparación médica y psicológica en los familiares del caso de Pueblo Bello e Ituango. Documento interno de trabajo. Bogotá: Corporación Vínculos. Recuperado de: https:// res.uniandes.edu.co/pdf/descargar. php?f=./data/Revista No 36/03 Dossier 02.pdf
Sistema de corresponsabilidad, (2013). Documento técnico para consulta. Equipo Interinstitucional de Asistencia Técnica Territorial. Disponible en: https://www. mininterior.gov.co/sites/ default/files/noticias/sc documentoconsulta.pdf

Sluzki, C. (1995). Violencia familiar y violencia política. En nuevos paradigmas, cultura y subjetividad, ed. Dora Fried Schnitman, 351-370. Buenos Aires: Paidós.

Unidad para la Atención y Reparación Integral a las Víctimas, (2012). Informe departamental de hechos victimizantes. Elaboración Red Nacional de Información-Grupo Análisis e Investigación. Recuperado de: http://rni.unidadvictimas.gov. $\mathrm{co/sites/default/files/Documentos/}$ Meta.pdf 